Veterinary Record (2005) 156, 669-673

C. R. Helps, BSc, PhD,

P. Lait, BSc,

T. Gruffydd-Jones,

BVetMed, PhD, DipECVIM,

MRCVS,

D. A. Harbour, BSc, $\mathrm{PhD}$,

University of Bristol,

Bristol BS40 5DU

A. Damhuis,

E. A. M. Graat, $\mathrm{MSc}, \mathrm{PhD}$,

Wageningen University, 6700 AH Wageningen,

The Netherlands

U. Björnehammar, DVM,

Smadjursklinik, 45150

Uddevalla, Sweden

D. Bolta, DVM,

C/Poeta Josep Cervera I

Grifol 8, 46013 Valencia,

Spain

C. Brovida, DVM,

Ospedale Veterinario

Anubi, 10024 Torino, Italy

L. Chabanne, DVM, PhD,

G. Ferrand, DVM,

Ecole Nationale Vétérinaire

de Lyon, 69280 Marcy

l'Etoile, France

H. Egberink, DVM, PhD,

Utrecht University,

3584 CL Utrecht,

The Netherlands

A. Fontbonne, DVM,

E. Malandain, DVM, MSc,

Ecole Nationale Vétérinaire

d'Alfort, 94704 Maisons-

Alfort Cedex, France

M. G. Pennisi, DVM, PhD,

Università degli Studi di

Messina, 98168 Messina,

Italy

D. Gunn-Moore, BSc,

BVM\&S, PhD, MRCVS,

Royal (Dick) School of

Veterinary Studies,

Roslin EH25 9GR

K. Hartmann,

ProfDrVetMed,

DrMedVetHabil,

DipECVIM-CA,

C. Stengel, DrMedVet,

Ludwig-Maximilians

Universität, 80539 Munich, Germany

H. Lutz, ProfDrFVH, FAMH, Universität Zürich,

CH-8057 Zürich,

Switzerland

K. Möstl, ProfDr,

Veterinärmedizinische

Universität Wien, Vienna, Austria

Correspondence to

Dr Gunn-Moore

\title{
Factors associated with upper respiratory tract disease caused by feline herpesvirus, feline calicivirus, Chlamydophila felis and Bordetella bronchiseptica in cats: experience from 218 European catteries
}

\author{
C. R. Helps, P. Lait, A. Damhuis, U. Buörnehammar, D. Bolta, C. Brovida, \\ L. Chabanne, H. Egberink, G. Ferrand, A. Fontbonne, M. G. Pennisi, \\ T. Gruffydd-Jones, D. Gunn-Moore, K. Hartmann, H. Lutz, E. Malandain, \\ K. Möstl, C. Stengel, D. A. Harbour, E. A. M. Graat
}

A full history of the management practices and the prevalence of upper respiratory tract disease (URTD) at 218 rescue shelters, breeding establishments and private households with five or more cats was recorded. Oropharyngeal and conjunctival swabs and blood samples were taken from 1748 cats. The prevalences of feline herpesvirus (FHV), feline calicivirus (FCV), Chlamydophila felis and Bordetella bronchiseptica were determined by PCR on swab samples. An ELISA was applied to determine the prevalence of antibodies to $B$ bronchiseptica. The rates of detection by PCR of each pathogen in the cats in catteries with and without ongoing URTD were, respectively, FHV 16 per cent and 8 per cent; FCV 47 per cent and 29 per cent; $C$ felis 10 per cent and 3 per cent; and $B$ bronchiseptica 5 per cent and 1.3 per cent; the seroprevalences of $B$ bronchiseptica were 61 per cent and 41 per cent, respectively. There was evidence that FHV, FCV and $B$ bronchiseptica played a role in URTD. The risk factors associated with the disease were less than excellent hygiene, contact with dogs with URTD, and larger numbers of cats in the cattery or household.

UPPER respiratory tract disease (URTD) ('cat flu') is still a common clinical problem in cats. The main primary pathogens include feline herpesvirus type 1 (FHV) and feline calicivirus (FCV) (Gaskell and Dawson 1994). The clinical signs in cats with FHV and/or FCV infection include sneezing, an ocular and nasal discharge, conjunctivitis, dyspnoea and coughing, and oral ulceration is frequently observed in cats with FCV. Cats that recover from FHV or FCV infection usually become carriers (Gaskell and Povey 1973, Wardley 1976, Gaskell and Dawson 1994).

Chlamydophila felis (previously known as Chlamydia psittaci var felis) is mainly an ocular (conjunctival) pathogen, which establishes chronic infections during which the organism is excreted for several months (Storz 1988). It has been isolated from approximately 30 per cent of cats with conjunctivitis (Shewen and others 1980) but has also been implicated in some cases of feline URTD (Sykes and others 1997). There is growing evidence that Bordetella bronchiseptica is widespread in the feline population and that it can also be involved in URTD in cats (McArdle and others 1994, Bergman and others 1997, Binns and others 1999, Pasmans and others 2001). Cats infected experimentally with $B$ bronchiseptica show signs of respiratory disease that are frequently indistinguishable from those caused by FHV and FCV (Jacobs and others 1993, Coutts and others 1996). Coughing does not appear to be a characteristic feature of $B$ bronchiseptica infection in cats, although it has been observed in some cases (Binns and others 1999). Cats recovering from experimental infection with $B$ bronchiseptica shed the bacteria intermittently without showing clinical signs (Coutts and others 1996).

Several studies have examined the prevalence in cats of each of the four pathogens, FHV, FCV, $C$ felis and $B$ bronchiseptica, and evaluated the factors that predispose them to infection (Wardley and others 1974, Coutts and others 1994, McArdle and others 1994, Gunn-Moore and others 1995,
Sykes and others 1997, Binns and others 1999, 2000). The aim of this international study was to identify and quantify the risk factors associated with the diagnosis of these pathogens in cats in multi-cat households with and without URTD.

\section{MATERIALS AND METHODS}

\section{Selection procedure and data collection}

Multi-cat households, defined as households with at least five cats, were selected in nine European countries: Austria, France, Germany, Italy, the Netherlands, Spain, Sweden, Switzerland and the UK. The study was designed as a case-control study. A 'case' household was one where at least one cat had URTD at the time of sampling, and a 'control' household was one with no URTD and where there had been no URTD in any of the cats during the previous six months. An effort was made to include an equal number of households with and without URTD in each country. A cat with URTD was defined as a cat with a history of and present signs of URTD, such as sneezing, coughing and/or an oculonasal discharge.

In each household selected, each cat, up to a maximum of 10 , was included. In the households with URTD, the diseased cats were sampled first. If there were fewer than 10 diseased cats, healthy cats were also sampled. The following details were recorded for each household: the type of household (breeding, private or rescue shelter), presence or history of URTD, country, vaccination status, level of hygiene, possibility for cats to go outdoors, transport of cats for breeding or exhibition, introduction of new cats and the occurrence of parturition and weaning, and the presence of dogs and/or other animals with URTD and the presence of any people with URTD.

Cats of all ages and breeds were eligible for inclusion, but cats that had been treated with antimicrobial agents within the previous three weeks or with corticosteroids or progesto- 
gens within six weeks were excluded. The selected cats underwent a clinical examination, and factors such as their sex, age, presence or history of URTD, vaccination status and clinical signs attributable to URTD, for example, coughing, sneezing, rhinitis/nasal discharge, an ocular discharge, conjunctivitis and respiratory effort, were recorded.

\section{Polymerase chain reaction}

Oropharyngeal and ocular samples were taken from each cat with plain cotton swabs (Medical Wire and Equipment), which were returned immediately after sampling to their covers. The swabs were kept on ice after sampling and then stored at $-20^{\circ} \mathrm{C}$ until they were analysed. A quantitative PCR was performed for the identification of FHV, FCV, $C$ felis and $B$ bronchiseptica. Nucleic acid was isolated using the DNeasy 96 Tissue kit (Qiagen). Swabs were placed in $200 \mu$ l phosphatebuffered saline, $200 \mu \mathrm{l}$ buffer AL and $20 \mu \mathrm{l}$ proteinase $\mathrm{K}$ and incubated at $70^{\circ} \mathrm{C}$ for 20 minutes; subsequent procedures were as described by the manufacturer. Primers and Taqman probes were designed using Primer 3 to detect the thymidine kinase gene of FHV, Orf1 of FCV, the major outer membrane protein of $C$ felis, and the FimA gene of B bronchiseptica, with the feline 28S rDNA gene as a positive control.

To reverse-transcribe the FCV RNA present in the nucleic acid samples to CDNA, the following reaction was set up on ice: $9.25 \mu \mathrm{l} 2 \times$ Platinum QRT-PCR Thermoscript (Invitrogen), $0.37 \mu \mathrm{l} 10 \mu \mathrm{M}$ FCV reverse primer, $0.38 \mu \mathrm{l}$ Thermoscript/ Platinum Taq mix and $8.5 \mu \mathrm{l}$ template nucleic acid. The reaction was incubated at $55^{\circ} \mathrm{C}$ for 20 minutes to allow reverse transcription and then at $85^{\circ} \mathrm{C}$ for five minutes to inactivate the Thermoscript. The four-way multiplex real-time PCR assay to detect FHV, FCV and $C$ felis and feline 28S rDNA was set up as follows: $12.5 \mu \mathrm{l} 2 \times$ Hotstartaq (Qiagen), $1.5 \mu \mathrm{l} 50 \mathrm{mM} \mathrm{mag-}$ nesium chloride, $100 \mathrm{nM}$ FHV primers and probe, $200 \mathrm{~nm}$ FCV primers and probe, $100 \mathrm{nM} \mathrm{C}$ felis primers and probe, $200 \mathrm{nM}$ feline $28 \mathrm{~S}$ primers and $100 \mathrm{nM}$ feline $28 \mathrm{~S}$ probe, $5 \mu \mathrm{l}$ CDNA/gDNA sample prepared as described above, and water to bring the volume to $25 \mu \mathrm{l}$. The reactions were incubated at $95^{\circ} \mathrm{C}$ for 15 minutes and then for 50 cycles of $95^{\circ} \mathrm{C}$ for $10 \mathrm{sec}-$ onds and $60^{\circ} \mathrm{C}$ for 60 seconds. Fluorescence was detected at $530,575,620$ and $680 \mathrm{~nm}$ at each annealing step at $60^{\circ} \mathrm{C}$. The singleplex real-time PCR to detect $B$ bronchiseptica was set up as follows: $12.5 \mu \mathrm{l} 2 \times$ Hotstartaq (Qiagen), $1.5 \mu \mathrm{l} 50 \mathrm{mM} \mathrm{mag-}$ nesium chloride, 200nM B bronchiseptica primers and 50nM $B$ bronchiseptica probe, $5 \mu$ gDNA and water to bring the volume to $25 \mu \mathrm{l}$. The reactions were incubated at $95^{\circ} \mathrm{C}$ for 15 minutes and then for 45 cycles of $95^{\circ} \mathrm{C}$ for five seconds and $60^{\circ} \mathrm{C}$ for 10 seconds. Fluorescence was detected at $530 \mathrm{~nm}$ at each annealing step at $60^{\circ} \mathrm{C}$. All the reactions were run in duplicate.

The following gene sequences were used: FHV thymidine kinase gene (accession number M26660), FCV strain CV Orf1 (accession number M86379), $C$ felis major outer membrane protein gene (accession number AF269258), B bronchiseptica FimA (accession number AF111796) and feline 28S rDNA (accession number AF353617); Table 1 gives the sequences of the primers and probes. All the primers and probes were synthesised by Cruachem. Fluorescently quenched probes were labelled with either 6-FAM, Hex, Texas Red or CY5 at the 5' end and the appropriate black hole quencher at the $3^{\prime}$ end.

A positive result was obtained when the fluorescence of a sample increased above the threshold fluorescence value, determined as 10 times the mean standard deviation of fluorescence over the baseline cycles. The threshold cycle number was the PCR cycle at which the sample fluorescence equalled the threshold fluorescence.

\section{Serology}

Only cats more than four months old were eligible for blood sampling; a blood sample was collected into an EDTA tube and

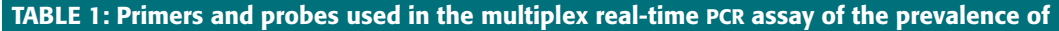
Teline calicivirus (FCV), feline herpesvirus (FHV), Chlamydophila felis and Bordetella
farlon bronchiseptica
brine calicivirus

\begin{tabular}{llc} 
Primer/Probe & Sequence (5'-3') & Region* \\
\hline FCV for & GTTGGATGAACTACCCGCCAATC & $2413-2435$ \\
FCV rev & CATATGCGGCTCTGATGGCTTGAAACTG & $2534-2507$ \\
FCV FQ & Hex TCGGTGTTTGATTGGCCTG BHQ1 & $2456-2475$ \\
FHV for & GGACAGCATAAAAGCGATTG & $173-192$ \\
FHV rev & AACGTGAACAACGACGCAG & $247-229$ \\
FHV FQ & CY5 AATTCCAGCCCGGAGCCTCAAT BHQ3 & $201-222$ \\
C felis for & GAACTGCAAGCAACACCACTG & $281-301$ \\
C felis rev & CCATTCGGCATCTTGAAGATG & $357-337$ \\
C felis FQ & FAM CGCTGCCACAGATCAAATTTGCC BHQ1 & $303-327$ \\
B bronchiseptica for & ACTATACGTCGGGAAATCTGTTG & $11,801-11,824$ \\
B bronchiseptica rev & CGTTGTCGCTTTCGTCTG & $11,881-11,863$ \\
B bronchiseptica FQ & FAM CGGGCCGATAGTCAGGGCGTAG BHQ1 & $11,830-11,851$ \\
Feline 28S rDNA for & AGCAGGAGGTGTGGAAGAG & $521-540$ \\
Feline 28S rDNA rev & AGGGAGAGCCTAAATCAAAGG & $620-600$ \\
Feline 28S rDNA FQ & Texas Red TGGCTTGTGGCAGCCAAGTGT BHQ2 & $557-577$ \\
\hline
\end{tabular}

* Numbers refer to the region of the gene where the primers or probes bind for Forward, rev Reverse, FQ Fluorescently quenched, BHQ Black hole quencher

the tubes were kept on ice while being transported. After centrifugation at room temperature, the plasma was transferred into a polypropylene tube (PPN-tube-96; Micronic) and stored at $-20^{\circ} \mathrm{C}$. An ELISA was applied to detect antibodies to $B$ bronchiseptica. Titres were calculated relative to a reference serum with a defined titre that was included in each ELISA plate (Jacobs and others 1993), and titres less than or equal to $2^{2}$ were considered negative.

An immunochromatographic test (DUO Speed; Bio Veto Test Laboratories) was used to detect feline leukaemia virus (FeLV) antigen and antibody to feline immunodeficiency virus (FIV).

\section{Statistical analysis}

Cats with antibodies to a pathogen were compared with cats without antibodies to that pathogen, and the individual cat was the statistical unit. The results are presented, where

\begin{tabular}{|c|c|c|c|}
\hline Variable & Classes & Fraction & Frequency (\%) \\
\hline \multirow[t]{2}{*}{ URTD in household } & URTD & $116 / 215$ & $54 \cdot 0$ \\
\hline & No URTD & $99 / 215$ & $46 \cdot 0$ \\
\hline \multirow[t]{3}{*}{ Type of cattery } & Breeding cattery & $106 / 207$ & $51 \cdot 2$ \\
\hline & Private household & $71 / 207$ & $34 \cdot 3$ \\
\hline & Rescue shelter & $30 / 207$ & $14 \cdot 5$ \\
\hline \multirow[t]{3}{*}{ Vaccination } & All cats & $150 / 206$ & $72 \cdot 8$ \\
\hline & Some of the cats & $33 / 206$ & $16 \cdot 0$ \\
\hline & None of the cats & $23 / 206$ & $11 \cdot 2$ \\
\hline \multirow[t]{2}{*}{ New cats } & New cats $<6 \mathrm{~m}$ & $92 / 214$ & $43 \cdot 0$ \\
\hline & No new cats $<6 \mathrm{~m}$ & $122 / 214$ & $57 \cdot 0$ \\
\hline \multirow[t]{2}{*}{ Hygiene } & Excellent & $85 / 204$ & 41.7 \\
\hline & Other & $119 / 204$ & $58 \cdot 3$ \\
\hline \multirow[t]{3}{*}{ Cats allowed outdoors } & All cats allowed outdoors & $78 / 184$ & $42 \cdot 4$ \\
\hline & Some cats allowed outdoors & 24/184 & $13 \cdot 0$ \\
\hline & No cats allowed outdoors & $82 / 184$ & $44 \cdot 6$ \\
\hline \multirow{2}{*}{$\begin{array}{l}\text { Transport cats for breeding } \\
\text { or exhibition }\end{array}$} & Transport $<6 \mathrm{~m}$ & $91 / 183$ & $49 \cdot 7$ \\
\hline & No transport $<6 \mathrm{~m}$ & $92 / 183$ & $50 \cdot 3$ \\
\hline \multirow[t]{2}{*}{ Parturition } & Parturition $<6 \mathrm{~m}$ & $90 / 178$ & $50 \cdot 6$ \\
\hline & No parturition <6m & $88 / 178$ & $49 \cdot 4$ \\
\hline \multirow[t]{2}{*}{ Weaning } & Weaning <6m & $74 / 178$ & 41.6 \\
\hline & No weaning $<6 \mathrm{~m}$ & 104/178 & $58 \cdot 4$ \\
\hline \multirow[t]{3}{*}{ Dog(s) present } & No dogs present & $98 / 212$ & $46 \cdot 2$ \\
\hline & Dogs present & $114 / 212$ & $53 \cdot 8$ \\
\hline & Dogs with URTD present & $6 / 114$ & $5 \cdot 3$ \\
\hline \multirow[t]{3}{*}{ Other animal(s) present } & No other animals present & 147/205 & 71.7 \\
\hline & Other animals present & $58 / 205$ & $28 \cdot 3$ \\
\hline & Other animals with URTD present & $2 / 58$ & $3 \cdot 4$ \\
\hline \multirow[t]{2}{*}{ People with URTD present } & No & 177/205 & $86 \cdot 3$ \\
\hline & Yes & $28 / 205$ & $13 \cdot 7$ \\
\hline
\end{tabular}

URTD Upper respiratory tract disease, $<6 \mathrm{~m}$ Within the past six months 
TABLE 3: Characteristics of the 1748 cats

\begin{tabular}{llcc} 
Variable & Classes & Fraction & Frequency (\%) \\
\hline URTD & URTD & $288 / 1712$ & $16 \cdot 8$ \\
\multirow{2}{*}{ URTD in household } & No URTD & $1424 / 1712$ & $83 \cdot 2$ \\
& URTD & $953 / 1713$ & $55 \cdot 6$ \\
Type of cattery & No URTD & $760 / 1713$ & $44 \cdot 4$ \\
& Breeding cattery & $933 / 1638$ & $57 \cdot 0$ \\
Sex & Private household & $505 / 1638$ & $30 \cdot 8$ \\
& Rescue shelter & $200 / 1638$ & $12 \cdot 2$ \\
& Entire male & $369 / 1664$ & $22 \cdot 2$ \\
Transport & Neutered male & $276 / 1664$ & $16 \cdot 6$ \\
\multirow{2}{*}{ Parturition and weaning } & Entire female & $746 / 1664$ & $44 \cdot 8$ \\
& Neutered female & $273 / 1664$ & $16 \cdot 4$ \\
& Transport $<6 \mathrm{~m}$ & $238 / 1687$ & $14 \cdot 1$ \\
& No transport $<6 \mathrm{~m}$ & $1449 / 1687$ & $85 \cdot 9$ \\
\hline
\end{tabular}

URTD Upper respiratory tract disease, $<6 \mathrm{~m}$ Within the previous six months

appropriate, as mean ( $\mathrm{sd}$ ). A univariate analysis was used to select variables for a multivariate analysis, with the level of significance $(\alpha)$ set at $0 \cdot 25$ for the univariate analysis and at 0.05 for the multivariate analysis. The results of the multivariate analysis are expressed as odds ratios (OR) with a 95 per cent confidence interval (CI). An or is calculated by dividing the odds in the exposed group by the odds in the non-exposed group; an OR of 1 indicates that there was no association between exposure and disease, an OR greater than 1 indicates that the exposed group has an increased risk compared with the unexposed group, and an OR less than 1 indicates that the exposed group has a decreased risk compared with the unexposed group.

First, a simple logistic regression (univariate analysis) was applied to each pathogen for each variable (PROC LOGISTIC, Release 8.2; SAS Institute), and the variables that were associated with the presence of a pathogen in an individual cat $(\mathrm{P}<0 \cdot 25)$ were used in the multivariate logistic regression. In the multivariate model, variables were excluded from the model by a backward elimination procedure according to the method of Hosmer and Lemeshow (1989). The least significant variable (based on Wald's statistic) was deleted, the model was refitted and the results were then compared (both the parameter estimates and the difference in -2 log likelihood of the model) with those of the previous run to check for confounding. With a change in parameter estimates $(\beta)$ of more than 25 per cent, or $0 \cdot 1$ if $\beta$ is between $-0 \cdot 4$ and $0 \cdot 4$, the deleted variable was considered to be a confounder and included in the model again. This resulted in a model containing variables related to the presence of a pathogen.

For the multivariate model, the number of cats available for the analysis was reduced greatly owing to the values for some variables being missing. In this model interaction effects were tested one by one. All the interactions that were significantly related $(\mathrm{P}<0 \cdot 05)$ to the presence of (antibodies to) a pathogen were then re-entered into the multivariate model and interactions were excluded as described above (Hosmer

\section{TABLE 4: Factors associated with feline herpesvirus in 1354 cats}

\begin{tabular}{llccccc} 
Variable & Category & $\begin{array}{c}\text { Number (\%) } \\
\text { of cats }\end{array}$ & Prevalence (\%) & OR & 95 per cent Cl & P \\
\hline Health status & No URTD & $660(49)$ & 8 & $1.0^{*}$ & - & - \\
of cattery & URTD & $694(51)$ & 16 & 1.95 & $1.11-3.44$ & 0.0205 \\
Hygiene & Excellent & $590(44)$ & 7 & $1.0^{*}$ & - & - \\
& Other & $764(56)$ & 16 & 2.09 & $1.17-3.71$ & 0.0121 \\
FCV & No FCV & $843(62)$ & 8 & $1.0^{*}$ & - & - \\
& FCV & $511(38)$ & 18 & 1.62 & $1.02-2.58$ & 0.0401
\end{tabular}

* Reference category

URTD Upper respiratory tract disease, OR Odds ratio, CI Confidence interval, FCV Feline calicivirus and Lemeshow 1989). Because multiple measurements in each cattery cannot be regarded as independent units of observation, the association of factors with the outcome variable was corrected for the fact that the cats were clustered within a multi-cat household (PROC GENMOD/REPEATED SUBJECT=CATTERY/TYPE=EXCH; SAS). The exchangeable correlation structure fitted best and was used to account for withinhousehold variation.

\section{RESULTS}

\section{Characteristics of the population of cats}

A total of 1748 cats from 218 multicat households was included in the study. Because the information recorded on some of the questionnaires was incomplete, the details of the households and cats have been expressed as a proportion of the cases with the appropriate information available (Tables $2,3)$. Swabs for the detection of FHV, FCV, $C$ felis and B bronchiseptica were available from 1724 of the cats, and blood samples were taken from 1463 of them.

Most of the cats had been vaccinated against FHV and FCV (67 per cent, 1163 of 1728), but some of them had also been vaccinated against $C$ felis (25 per cent, 294 of 1163). Rabies, feline panleukopenia (FPL), feline infectious peritonitis (FIP) and/or FeLV vaccines had also been administered to some of the cats. Of the fully completed questionnaires, signs of URTD were found in 20 per cent ( 105 of 533) of the cats that had not been vaccinated against FHV and FCV, and in 15 per cent (175 of 1136) of the cats that had been vaccinated against FHV and FCV $(\mathrm{P}=0 \cdot 0289 ; \mathrm{OR}=0 \cdot 74 ; 95$ per cent $\mathrm{CI} 0 \cdot 57$ to $0 \cdot 97)$. An attenuated vaccine against FHV and FCV had been used in 683 of 1103 cats ( 62 per cent) and an inactivated vaccine had been used in the other 420 ( 38 per cent). Both vaccines reduced the prevalence of URTD and there was no significant difference in the prevalence of URTD between cats vaccinated with the attenuated and the inactivated vaccines $(\mathrm{P}=0 \cdot 2535)$.

FeLV antigen was present in the blood samples from 4.2 per cent of the cats with URTD and in 1.2 per cent of the cats without URTD ( $\mathrm{P}=0 \cdot 0014 ; \mathrm{OR}=3 \cdot 70,95$ per cent $\mathrm{CI} 1 \cdot 66$ to $8 \cdot 24)$. FIV antibodies were present in the blood of $7 \cdot 7$ per cent of the cats with URTD and in 3.8 per cent of the cats without URTD $(\mathrm{P}=0 \cdot 0068 ; \mathrm{OR}=2 \cdot 12,95$ per cent $\mathrm{CI} 1 \cdot 23$ to $3 \cdot 66)$.

Of the 218 households involved in the study, there were 50 (23 per cent) in France, 37 (17 per cent) in Italy, 37 in Germany, 23 (10 per cent) in the UK, 21 (10 per cent) in the Netherlands, 21 in Switzerland, 13 (6 per cent) in Spain, nine in Austria and seven in Sweden. Three households, with 60, 100 and 109 cats, were excluded from the multivariate analysis because they skewed the results. The mean (sd) number of cats in the other 215 households was $12(7 \cdot 3)$ with a median of 10 , and a range from five to 45 cats. The mean age of 1713 of the cats was $3.6(3.4)$ years, with a median of 2.6 years, and a range from 10 days to 20 years.

\section{Multivariate analysis of FHV}

The results of the multivariate analysis of FHV are shown in Table 4. In the catteries with URTD, the prevalence of FHV was twice as high (16 per cent) as in the catteries with no URTD (8 per cent) $(\mathrm{P}=0 \cdot 0205 ; \mathrm{OR}=1 \cdot 95,95$ per cent $\mathrm{CI} 1 \cdot 11$ to $3 \cdot 44)$. A less than excellent level of hygiene in a cattery increased the prevalence of $\mathrm{FHV}(\mathrm{P}=0 \cdot 0121 ; \mathrm{OR}=2 \cdot 09,95$ per cent $\mathrm{CI} 1 \cdot 17$ to $3 \cdot 71)$. A higher proportion of the cats that were infected with FCV were positive for FHV (18 per cent) than of the cats that were not infected with FCV ( 8 per cent) $(\mathrm{P}=0 \cdot 0401 ; \mathrm{OR}=1 \cdot 62$, 95 per cent CI 1.02 to $2 \cdot 58$ ).

\section{Multivariate analysis of FCV}

The results of the multivariate analysis of FCV are shown in Table 5. The catteries with URTD had a higher proportion of 
FCV-positive cats ( 47 per cent) than the catteries with no URTD (29 per cent) $(\mathrm{P}=0 \cdot 0023 ; \mathrm{OR}=1 \cdot 89,95$ per cent $\mathrm{CI} 1 \cdot 25$ to $2 \cdot 84)$. Less than excellent hygiene increased the prevalence of FCV $(\mathrm{P}=0 \cdot 0260 ; \mathrm{OR}=1 \cdot 60,95$ per cent $\mathrm{CI} 1 \cdot 06$ to $2 \cdot 41)$. The prevalence of FCV infection was lower in the catteries where one or more dogs were present than in the catteries with no dogs $(\mathrm{P}=0 \cdot 0134 ; \mathrm{OR}=0 \cdot 60,95$ per cent $\mathrm{CI} 0 \cdot 41$ to $0 \cdot 90)$. $\mathrm{FCV}$ was detected in a higher proportion of the cats with FHV infection (57 per cent) than of the cats with no FHV infection ( 35 per cent $)(\mathrm{P}=0 \cdot 0097 ; \mathrm{OR}=1 \cdot 59,95$ per cent $\mathrm{CI} 1 \cdot 12$ to $2 \cdot 25)$.

\section{Multivariate analysis of $\mathrm{C}$ felis}

The results of the multivariate analysis of $C$ felis are shown in Table 6. A higher proportion of the cats in the catteries with URTD were infected with $C$ felis (10 per cent) than of the cats in the catteries with no URTD ( 3 per cent $)(\mathrm{P}=0 \cdot 0502 ; \mathrm{OR}=2 \cdot 55$, 95 per cent $\mathrm{CI} 1.00$ to 6.53$)$. C felis was detected in a higher proportion of the cats in catteries with less than excellent hygiene $(\mathrm{P}=0 \cdot 0490 ; \mathrm{OR}=3 \cdot 09,95$ per cent $\mathrm{CI} 1 \cdot 01$ to $9 \cdot 51)$. A smaller proportion of the cats in catteries with dogs were infected with $C$ felis than of those in catteries with no dogs $(\mathrm{P}=0 \cdot 0106 ; \mathrm{OR}=0 \cdot 27,95$ per cent $\mathrm{CI} 0 \cdot 10$ to $0 \cdot 74)$.

\section{Multivariate analysis of $B$ bronchiseptica}

The results of the multivariate analysis of $B$ bronchiseptica are shown in Table 7. A higher proportion of the cats in catteries with URTD were infected with $B$ bronchiseptica ( 5.0 per cent) than of those in catteries with no URTD ( 1.3 per cent) $(\mathrm{P}=0 \cdot 0012 ; \mathrm{OR}=3 \cdot 50,95$ per cent $\mathrm{CI} 1 \cdot 64$ to $7 \cdot 46)$. The more cats there were in the cattery, the greater the likelihood that $B$ bronchiseptica was detected $(\mathrm{P}=0 \cdot 0307 ; \mathrm{OR}=1 \cdot 05,95$ per cent CI 1.00 to $1 \cdot 11)$.

\section{Multivariate analysis of $B$ bronchiseptica serology}

The results of the multivariate analysis of $B$ bronchiseptica antibodies are shown in Table 8. A higher proportion of the cats that lived in catteries with URTD had antibodies to $B$ bronchiseptica (61 per cent) than of the cats in catteries with no URTD ( 41 per cent $)(\mathrm{P}=0 \cdot 0026 ; \mathrm{OR}=2 \cdot 00,95$ per cent $\mathrm{CI} 1 \cdot 27$ to $3 \cdot 14)$. A less than excellent level of hygiene in a cattery increased the prevalence of cats with antibodies to $B$ bronchiseptica ( $\mathrm{P}=0.0211$; OR=1.73, 95 per cent $\mathrm{CI} 1.09$ to $2 \cdot 74)$. A significantly higher proportion of the cats in rescue shelters (77 per cent) were antibody-positive for $B$ bronchiseptica than of the cats in private households ( 53 per cent) or breeding catteries ( 46 per cent) $(\mathrm{P}=0 \cdot 0123 ; \mathrm{OR}=0 \cdot 38,95$ per cent $\mathrm{Cl} 0 \cdot 17$ to $0 \cdot 81$, and $\mathrm{P}=0 \cdot 0059$; $\mathrm{OR}=0 \cdot 36,95$ per cent $\mathrm{CI} 0 \cdot 17$ to $0 \cdot 74$, respectively). Advancing age increased the risk of cats being $B$ bronchiseptica antibody-positive by 1.09 times for each year of age $(\mathrm{P}<0.0001 ; 95$ per cent $\mathrm{CI} 1.06$ to $1 \cdot 13)$.

\section{DISCUSSION}

The prevalence of FCV, FHV and B bronchiseptica, but not $C$ felis, was significantly higher in the multi-cat households with active URTD than in the multi-cat households without URTD, suggesting that each of these three pathogens plays a role in the disease. The prevalences of FHV and FCV in the catteries with URTD ( 16 per cent and 47 per cent, respectively) and in those with no URTD ( 8 per cent and 29 per cent, respectively) were slightly higher than the prevalences reported previously (Wardley and others 1974, Coutts and others 1994, Binns and others 2000). This difference may have been due to the sampling criteria employed, but it may also be explained by the higher sensitivity of the PCR used in the present study than the virus isolation techniques used previously. The fact that carrier cats shed FCV almost continuously, whereas FHV is shed only intermittently between periods of latency,

\begin{tabular}{|c|c|c|c|c|c|c|}
\hline Variable & Category & $\begin{array}{l}\text { Number (\%) } \\
\text { of cats }\end{array}$ & Prevalence (\%) & OR & 95 per cent $\mathrm{Cl}$ & $P$ \\
\hline \multirow{2}{*}{$\begin{array}{l}\text { Health status } \\
\text { of cattery }\end{array}$} & No URTD & 664 (49) & 29 & $1.0^{*}$ & - & - \\
\hline & URTD & 697 (51) & 47 & 1.89 & $1 \cdot 25-2 \cdot 84$ & 0.0023 \\
\hline \multirow[t]{2}{*}{ Hygiene } & Excellent & 578 (42) & 29 & $1.0^{*}$ & - & - \\
\hline & Other & 783 (58) & 45 & 1.60 & $1.06-2.41$ & 0.0260 \\
\hline \multirow[t]{2}{*}{ Dog(s) present } & No & $656(48)$ & 44 & $1.0^{*}$ & - & - \\
\hline & Yes & 705 (52) & 33 & 0.60 & $0.41-0.90$ & 0.0134 \\
\hline \multirow[t]{2}{*}{ FHV } & No FHV & 1204 (88) & 35 & $1.0^{*}$ & - & - \\
\hline & FHV & 157 (12) & 57 & 1.59 & $1 \cdot 12-2 \cdot 25$ & 0.0097 \\
\hline
\end{tabular}

* Reference category

URTD Upper respiratory tract disease, OR Odds ratio, CI Confidence interval, FHV Feline herpesvirus

\begin{tabular}{|c|c|c|c|c|c|c|}
\hline Variable & Category & $\begin{array}{l}\text { Number (\%) } \\
\text { of cats }\end{array}$ & Prevalence (\%) & OR & 95 per cent $\mathrm{Cl}$ & $\mathrm{P}$ \\
\hline \multirow{2}{*}{$\begin{array}{l}\text { Health status } \\
\text { of cattery }\end{array}$} & No URTD & $558(51)$ & 3 & $1.0 *$ & - & - \\
\hline & URTD & 543 (49) & 10 & 2.55 & $1 \cdot 00-6.53$ & 0.0502 \\
\hline \multirow[t]{2}{*}{ Hygiene } & Excellent & $470(43)$ & 2 & $1.0^{*}$ & - & - \\
\hline & Other & 631 (57) & 9 & 3.09 & $1.01-9.51$ & 0.0490 \\
\hline \multirow{2}{*}{ Dog(s) present } & No & $498(45)$ & 10 & $1.0 *$ & - & - \\
\hline & Yes & $603(55)$ & 3 & 0.27 & $0.10-0.74$ & 0.0106 \\
\hline
\end{tabular}

* Reference category

URTD Upper respiratory tract disease, OR Odds ratio, CI Confidence interval

explains the generally higher isolation rate of FCV compared with FHV (Gaskell and Povey 1973, Wardley 1976, Gaskell and Dawson 1994, Binns and others 2000).

$B$ bronchiseptica was detected in only 5 per cent of the cats with URTD and in 1.3 per cent of those without URTD. These low figures probably underestimate the significance of this pathogen in URTD in cats, because its seroprevalence indicates that the infection is widespread. Moreover, the OR suggests that the cats in households with URTD were three times more likely to have B bronchiseptica than cats in households without URTD; the risk also increased as the number of cats in the household increased.

During the study a PCR was developed to detect $B$ bronchiseptica infection. The PCR has advantages over bacterial or viral isolation and is less time-consuming, less expensive and, most importantly for a study of this type, imposes fewer restrictions in terms of transport and logistics. However, the PCR method was found to be up to 10 times less sensitive than bacterial culture, owing to technical constraints with the DNA extraction step, and it was unable to detect fewer than 100 pathogens per swab.

The results of this study provide further evidence that $B$ bronchiseptica is widespread in multi-cat households (McArdle and others 1994, Bergman and others 1997) and is associated with clinical signs of URTD in cats (Binns and others 1999, Pasmans and others 2001). The long-term asymptomatic carriage of B bronchiseptica (Coutts and others 1996) and its association with stress (Pedersen 1988) have been reported previously. It is possible that in the field carrier cats may remain culture-negative except during periods of stress. These findings underline the difficulty faced by veterinarians when dealing with feline URTD in clinical practice in assessing whether B bronchiseptica may be one of the causal agents.

Less than excellent hygiene was associated with an increase in the prevalence of FHV, FCV and $C$ felis and of antibodies to $B$ bronchiseptica. FHV is rather unstable in the environment but FCV can survive for eight to 10 days (Dawson and Willoughby 1999). B bronchiseptica can survive long enough outside the host to allow it to be transmitted within infected mucus (Speakman and others 1999), especially in a heavily contaminated environment. 
TABLE 7: Factors associated with Bordetella bronchiseptica in 1527 cats

\begin{tabular}{llccccc} 
Variable & Category & $\begin{array}{c}\text { Number }(\%) \\
\text { of cats }\end{array}$ & Prevalence (\%) & OR & 95 per cent Cl & P \\
\hline Health status & No URTD & $690(45)$ & 1.3 & 3.50 & $1.64-7.46$ & 0.0012 \\
$\begin{array}{l}\text { of cattery } \\
\text { Number }\end{array}$ & URTD & $837(55)$ & 5.0 & & & \\
\hline
\end{tabular}

URTD Upper respiratory tract disease, OR Odds ratio, CI Confidence interval

\section{TABLE 8: Factors associated with Bordetella bronchiseptica antibodies in 1229 cats}

\begin{tabular}{|c|c|c|c|c|c|c|}
\hline Variable & Category & $\begin{array}{l}\text { Number (\%) } \\
\text { of cats }\end{array}$ & Prevalence (\%) & OR & 95 per cent $\mathrm{Cl}$ & $\mathrm{P}$ \\
\hline \multirow{2}{*}{$\begin{array}{c}\text { Health status } \\
\text { of cattery }\end{array}$} & No URTD & $541(44)$ & 41 & $1.0^{*}$ & & \\
\hline & URTD & $688(56)$ & 61 & $2 \cdot 00$ & $1 \cdot 27-3 \cdot 14$ & 0.0026 \\
\hline \multirow[t]{2}{*}{ Hygiene } & Excellent & 509 (41) & 41 & $1.0^{*}$ & - & - \\
\hline & Other & 720 (59) & 60 & 1.73 & $1.09-2.74$ & 0.0211 \\
\hline \multirow[t]{3}{*}{ Type of cattery } & Private household & $364(30)$ & 53 & 0.38 & $0 \cdot 17-0.81$ & 0.0123 \\
\hline & Breeding cattery & 710 (58) & 46 & 0.36 & $0.17-0.74$ & 0.0059 \\
\hline & Rescue shelter & 155 (13) & 77 & $1.0^{*}$ & - & - \\
\hline \multirow{3}{*}{$\begin{array}{l}\text { Mean (sd) age } \\
\text { of cats (years) }\end{array}$} & $3.9(3.4)$, median & & & & & \\
\hline & $3 \cdot 0$, range three & & & & & \\
\hline & weeks to 20 years & & 52 & 1.09 & $1.06-1.13<$ & $<0.0001$ \\
\hline
\end{tabular}

* Reference category

URTD Upper respiratory tract disease, OR Odds ratio, CI Confidence interval

Previous work has shown that contact with dogs with URTD can be a risk factor for URTD in cats (Binns and others 1998, 1999, Dawson and others 2000). However, in this study, the presence of a dog decreased the risk of detection of FCV and/or $C$ felis, the first time that this relationship has been observed. It may be an indirect effect related to the type of cattery, because there were far fewer rescue shelters with dogs (31 per cent) than breeding catteries (57 per cent) or private households (59 per cent). In the univariate analysis the prevalence of cats with antibodies to $B$ bronchiseptica was higher in multi-cat households in which dogs were present (data not shown), suggesting that the pathogen was being transmitted between dogs and cats, as reported previously by Dawson and others (2000). This variable was no longer significant in the multivariate analysis, probably owing to the small number of dogs with URTD that were involved in the study. It is perhaps not surprising that cats in rescue shelters were at a higher risk of URTD than cats in breeding catteries or private households. Moreover, the larger the number of cats the greater the risk, particularly for $B$ bronchiseptica.

The recognition of acute infectious URTD in cats is usually straightforward, but the identification of the underlying infectious agents involved can be difficult, particularly when two (FCV and FHV) and sometimes three (FCV, FHV and B bronchiseptica) causal agents are present. This is particularly the case when $B$ bronchiseptica is involved, because no definitive diagnostic test for this agent is readily available. The high prevalence of antibodies to B bronchiseptica in the present study clearly indicates that infection with this organism is widespread in European cats. The PCR, in spite of its limitations, showed that this pathogen plays a significant role in active URTD, particularly in larger multi-cat households. Minimising the risk factors identified in this study and optimising vaccination programmes in multi-cat households can contribute to reducing URTD. The inclusion of the novel intranasal vaccine against $B$ bronchiseptica (Williams and others 2002) into vaccination programmes may help to reduce the prevalence of URTD in some multi-cat households.

\section{ACKNOWLEDGEMENTS}

The authors are grateful to N. de Schwartz for coordinating the study, and to A. Gray and L. Horspool for their useful comments on the manuscript. In addition, they would like to thank A. Jacobs and P. Vermeij for their contribution to the sequencing of feline Bordetella FimA.

\section{References}

BERGMAN, J. G. H. E., VERNOOIJ, J. \& ZEGERS, E. M. (1997) Prevalence of antibodies against Bordetella bronchiseptica in cats with a history of respiratory disease. Veterinary Quarterly 19 (Suppl 1), S50-S51

BINNS, S. H., DAWSON, S., SPEAKMAN, A. J., CUEVAS, L. E, GASKELL, C. J., HART, C. A., MORGAN, K. L. \& GASKELL, R. M. (1999) Prevalence and risk factors for feline Bordetella bronchiseptica infection. Veterinary Record 144, 575-580

BINNS, S. H., DAWSON, S., SPEAKMAN, A. J., CUEVAS, L. E., GASKELL, C. J., HART, C. A., MORGAN, K. L. \& GASKELL, R. M. (2000) A study of feline upper respiratory tract disease with reference to prevalence and risk factors for infection with feline calicivirus and feline herpesvirus. Journal of Feline Medicine and Surgery 2, 123-133

BINNS, S. H., SPEAKMAN, A. J., DAWSON, S., BENNETT, M., GASKELL R. M. \& HART, C. A. (1998) The use of pulsed-field gel electrophoresis to examine the epidemiology of Bordetella bronchiseptica isolated from cats and other species. Epidemiology and Infection 120, 201-208

COUTTS, A. J., DAWSON, S., BINNS, S., HART, C. A., GASKELL, C. J. \& GASKELL, R. M. (1996) Studies on natural transmission of Bordetella bronchiseptica in cats. Veterinary Microbiology 48, 19-27

COUTTS, A. J., DAWSON, S., WILLOUGHBY, K. \& GASKELL, R. M. (1994) Isolation of feline respiratory viruses from clinically healthy cats at UK cat shows. Veterinary Record 135, 555-556

DAWSON, S., JONES, D., MCCRACKEN, C. M., GASKELL, R. M., HART, C. A. \& GASKELL, C. J. (2000) Bordetella bronchiseptica infection in cats following contact with infected dogs. Veterinary Record 146, 46-48

DAWSON, S. \& WILLOUGHBY, K. (1999) Feline infectious upper respiratory tract disease - an update. In Practice 21, 232-237

GASKELL, R. M. \& DAWSON, S. (1994) Viral-induced upper respiratory tract disease. In Feline Medicine and Therapeutics. 2nd edn. Oxford, Blackwell. pp 453-472

GASKELL, R. M. \& POVEY, R. C. (1973) Re-excretion of feline viral rhinotracheitis virus following corticosteroid treatment. Veterinary Record 93, 204-205

GUNN-MOORE, D. A., WERRETT, G., HARBOUR, D. A., FEILDEN, H. \& GRUFFYDD-JONES, T. J. (1995) Prevalence of Chlamydia psittaci antibodies in healthy pet cats in Britain. Veterinary Record 136, 366-367

HOSMER, D. W. \& LEMESHOW, S. (1989) Model-building strategies and methods for logistic regression. In Applied Logistic Regression. New York, John Wiley and Sons. pp 82-134

JACOBS, A. A. C., CHALMERS, W. S. K., PASMAN, J., VAN VUGT, F. \& CUENEN, L. H. (1993) Feline bordetellosis: challenge and vaccine studies. Veterinary Record 133, 260-263

MCARDLE, H. C., DAWSON, S., COUTTS, A. J., BENNETT, M., HART, C. A RYVAR, R. \& GASKELL, R. M. (1994) Seroprevalence and isolation rate of Bordetella bronchiseptica in cats in the UK. Veterinary Record 134, 506507

PASMANS, F., ACKE, M., VANROBAEYS, M. \& HAESEBROUCK, F. (2001) Prevalence of Bordetella bronchiseptica infections in cats from different environments. Vlaams Diergeneeskundig Tijdschrift 70, 124-126

PEDERSEN, N. (1988) Bordetellosis. In Feline Infectious Disease. St Louis, Mosby. pp 153-154

SHEWEN, P. E., POVEY, R. C. \& WILSON, M. R. (1980) A survey of the conjunctival flora of clinically normal cats and cats with conjunctivitis. Canadian Veterinary Journal 21, 231-233

SPEAKMAN, A. J., DAWSON, S., BINNS, S. H., GASKELL, C. J., HART, C. A. \& GASKELL, R. M. (1999) Bordetella bronchiseptica infection in the cat Journal of Small Animal Practice 40, 252-256

STORZ, J. (1988) Overview of animal diseases induced by chlamydial infection. In Microbiology of Chlamydia. Ed A. L. Barron. Boca Raton, CRC Press. pp 167-192

SYKES, J. E., STUDDERT, V. P., ANDERSON, G. \& BROWNING, G. F. (1997) Comparison of $C$ felis from cats with upper respiratory tract disease by polymerase chain reaction analysis of the ompA gene. Veterinary Record 140, 310-313

WARDLEY, R. C. (1976) Feline calicivirus carrier state. A study of the host/virus relationship. Archives of Virology 52, 243-249

WARDLEY, R. C., GASKELL, R. M. \& POVEY, R. C. (1974) Feline respiratory viruses - their prevalence in clinically healthy cats. Journal of Small Animal Practice 15, 570-586

WILLIAMS, J., LARIS, R., GRAY, A. W. \& JACOBS, A. A. C. (2002) Studies of the efficacy of a novel intranasal vaccine against feline bordetellosis. Veterinary Record 150, 439-442 